\title{
Finite-size scaling on the Ising coexistence line*
}

\author{
Sourendu Gupta ${ }^{\mathrm{a}}$ and A. Irbäck ${ }^{\mathrm{b}}$

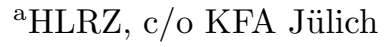 \\ D-5170 Jülich, Germany \\ ${ }^{\mathrm{b}}$ Theory Division, CERN \\ CH-1211 Geneva 23, Switzerland
}

We study the finite-size scaling of moments of the magnetization in the low-temperature phase of the twodimensional Ising model.

\section{INTRODUCTION}

Recently, finite-size scaling (FSS) at first-order phase transitions has attracted considerable interest. Its practical importance in connection with Monte Carlo studies has become increasingly clear, for instance through the controversy about the order of the phase transition in quenched QCD. At the same time, rigorous developments have been accomplished by Borgs, Kotecký and Miracle-Solé [1.22]. These results were obtained for a class of models that includes the Ising model at large $\beta$ and $q$-state Potts models with large $q$, and were proposed to be more general. The FSS model suggested by the results is quite powerful. It provides, for instance, an estimator of the transition coupling that should have exponentially small finite-size corrections. Numerical studies of Potts models support this prediction 3,4], but have also found less convincing agreement for some other quantities [4,5]. To find out more about the applicability of the FSS model we have performed a numerical study of the twodimensional Ising model [6].

For this model, the results of ref. [1] show that the partition function in a large periodic box $L^{d}$, at large $\beta$, is well approximated by

$Z(h, L) \approx e^{L^{d} f_{+}(h)}+e^{L^{d} f_{-}(h)}$,

where $h=\beta H, H$ being the external magnetic field. The functions $f_{ \pm}(h)$ are related to the freeenergy density $f(h)=\lim _{L \rightarrow \infty} L^{-d} \ln Z(h, L)$ by

\footnotetext{
*Presented by A. Irbäck
}

$f_{+(-)}(h)>f_{-(+)}(h)=f(h)$ if $h>0(<0)$, and $f_{+}(0)=f_{-}(0)$. Each term in eq. (11) represents fluctuations about one of the two coexisting phases, whereas the omitted remainder accounts for mixed configurations and is exponentially suppressed in $L$. This decomposition is the starting point for the finite-size analysis of moments of the order parameter. One proceeds by expanding the functions $f_{ \pm}(h)$ about $h=0$ and obtains, at fixed $h L^{d}$, expansions of the moments in powers of $L^{-d}$. The first-order finite-size corrections, to combinations of moments of up to fourth order, have been investigated numerically for the $q$-state Potts model with $q=7,8$ and 10 in refs. [4, 5]. As already alluded to, the agreement was not perfect, indicating that the asymptotic $L$ regime had not been reached. First-order corrections have also been analysed numerically for the Ising model [7], within the framework of the double-Gaussian model [7] 8]. In this earlier phenomenological FSS model, the two peaks in the probability distribution for the order parameter were approximated by Gaussians. This corresponds to a quadratic approximation for $f_{ \pm}$in the model discussed here, as can be seen by inverse Laplace transformation. As a result, the double-Gaussian model produces the same first-order expressions, except for problems with the relative normalization of the peaks. For the Ising model the normalization problem is absent thanks to the global spin-flip symmetry.

We shall extend these considerations in two directions: we shall study the size-dependence beyond the first-order level and we shall include in 
the analysis moments of higher order.

\section{HIGHER ORDERS}

We consider the behaviour of the moments at the phase transition. We calculate FSS expressions from eq. (1), assuming that the contribution from the omitted tunnelling term is exponentially suppressed. For definiteness, we restrict ourselves to the Ising case. The power-like volume dependence arises from fluctuations about the two stable phases and is obtained by expanding the functions $f_{ \pm}$about $h=0$. We write

$f_{+}(h)=\sum_{k=0}^{\infty} \frac{a_{k}}{k !} h^{k}$,

and, by symmetry, the corresponding coefficients for $f_{-}$then are $(-1)^{k} a_{k}$. The coefficient $a_{1}=m_{0}$ is the spontaneous magnetization and $\beta a_{2}=\chi$ the susceptibility. In order to obtain the moment $\left\langle m^{k}\right\rangle$ at $h=0$ it is sufficient to consider terms up to $k$ th order in the expansions of $f_{ \pm}$. Inserting these expansions into eq. (11), it is easily found, for even $k(>0)$, that

$$
\begin{aligned}
& \left\langle m^{k}\right\rangle=a_{1}^{k}+L^{-d} \theta_{k 2}\left(\begin{array}{l}
k \\
2
\end{array}\right) a_{2} a_{1}^{k-2}+L^{-2 d} \times \\
& {\left[\theta_{k 3}\left(\begin{array}{c}
k \\
3
\end{array}\right) a_{3} a_{1}^{k-3}+\theta_{k 4} 3\left(\begin{array}{l}
k \\
4
\end{array}\right) a_{2}^{2} a_{1}^{k-4}\right]+\ldots}
\end{aligned}
$$

where $\theta_{i j}=1$ if $i \geq j$ and 0 otherwise. The coefficient in front of $L^{-j d}$ is a function of $a_{1}, \ldots, a_{j+1}$, and it is zero if $j \geq k$. Odd moments, of course, vanish becuase of the spin-flip symmetry.

For moments of moderate order $k$ it is straightforward to extend eq. (3) so as to include all the power-like corrections. The validity of the resulting expressions will however depend on $k$. In general we expect that the (approximate) validity gets restricted to larger $L$ with increasing $k$. Note that higher coefficients in eq. (3) can be large not only because of the combinatorial factors, but also because they involve higher $a_{k}$. The series eq. (2) is asymptotic only [9].

Having obtained the prediction, we next discuss how to apply and test it. For the twodimensional Ising model, the spontaneous magnetization $a_{1}$ is exactly known, and fairly precise estimates of higher $a_{k}$ 's exist in the literature. Thus, one has a parameter-free prediction for loworder moments. In general, the $a_{k}$ 's are, however, unknown. We may then try to use fits of numerical data to eq. (3) to extract them. By checking for consistency values obtained from different moments, a test of the FSS model is obtained. In practice, such a program is not easy to follow, and we are therefore led to look for other possibilities. An obvious one is to try to use singlephase cumulants. We define the measurement of the single-phase cumulant by introducing a cut between the two peaks in the probability distribution for the order parameter. In the remaining part, say $m>m^{*}$, where $\left|m^{*}\right|<m_{0}$, we expect contributions from the disfavoured phase, as defined by the decomposition of eq. (11), as well as from mixed phases, to be exponentially suppressed in $L$. Up to exponentially small errors, we therefore expect

$a_{k}=L^{(k-1) d}\left\langle m^{k}\right\rangle_{c}^{*}$,

where the ${ }^{*}$ indicates expectation value over $m>$ $m^{*}$. In our calculations we take $m^{*}=0$, but in principle one could choose any $\left|m^{*}\right|<m_{0}$. Note also that the applicability of eq. (4) depends on $k$. The measurement of $\left\langle m^{k}\right\rangle_{c}^{*}$ rapidly becomes more difficult with increasing order $k$, since it is $O\left(L^{-(k-1) d}\right)$ and is the result of cancellations between $O(1)$ numbers.

\section{NUMERICAL RESULTS}

We now would like to test the finite-size expression for moments, eq. (3), and also the feasibility of a numerical determination of the coefficients $a_{k}$ through eq. (4). To this end we have performed numerical simulations of the twodimensional Ising model. These were done at $u / u_{c}=0.9$, where $u=\exp (-4 \beta)$ and $u_{c}=$ $3-2 \sqrt{2}$. At this temperature, the exactly known values of the correlation length and the spontaneous magnetisation are $\xi \approx 4.8$ and $m_{0} \approx 0.84$, respectively. In addition, several of the coefficients $a_{k}$ are known to high precision from powerseries expansions [9].

Using the Swendsen-Wang cluster algorithm, we simulated $L^{2}$ lattices with $16 \leq L \leq 60$. For 
each size, 4 million iterations were done. Measurements were separated by 10 iterations and errors were estimated through a jackknife procedure.

The first three (normalized) single-phase cumulants $c_{k}=L^{(k-1) d}\left\langle m^{k}\right\rangle_{c}^{*}$ are shown in fig. 1. They were obtained by folding the probability distribution onto $m>0$. From the above discussion, we expect that $c_{k}$ approaches $a_{k}$ at an exponential rate for large $L$. While our data at large $L$ are consistent with such a behaviour, it is clear that the smaller $L$ studied do not belong to the asymptotic region. Unfortunately, the data points left are insufficient for a study of the form of the asymptotic $L$ dependence. On the other hand, the close agreement found at large $L$ with the values in ref. [9] shows that good estimates of the infinite volume limit can be obtained from the range of $L$ studied. For instance, we note that the first and second cumulants are only a few per cent off their infinite volume values if $L / \xi>5$. It would therefore be possible to determine the $L^{-d}$ term of the moments to a similar accuracy.

In fig. 2 we compare our results for the first three even moments with the prediction of eq. (3), using values of the $a_{k}$ 's from ref. [9]. For each moment all non-vanishing terms in the expansion have been included. The curve labelled $j$ is the result obtained by including the terms of order up to $L^{-j d}$. At large $L$, the prediction is in very good agreement with the data, and the agreement is improved by adding more terms. The extent of this asymptotic $L$ region already shows, for these three moments, a dependence on the order of the moment, as discussed above. Larger $L$ is required for the sixth moment. For the second and fourth moment the difference between measured and predicted finite-size correction is less than $10 \%$ down to the smallest size studied, $L / \xi \approx 3.3$. The largest discrepancy for the second moment, at $L / \xi \approx 3.3$, is $6 \%$ or $3.4 \sigma$.

\section{SUMMARY}

We have studied the finite-size dependence of moments of the magnetization in the twodimensional Ising model. The FSS model by Borgs, Kotecký and Miracle-Solé gives a very
Figure 1. Normalized single-phase cumulants $c_{k}$ against $L^{-d}$. The horizontal lines are the coefficients $a_{k}$, as given by ref. [9]. 
good description of the large- $L$ results. Data for the first three even moments showed, in fact, no significant deviation for $L / \xi>6$, after taking higher-order corrections into account. The description remains approximately valid at the smaller $L / \xi>3.3$ studied for the second and fourth moment, but not for the sixth. Existing analytical results allowed us to perform these tests without using any free parameters. In general the parameters of the FSS model are unknown and we therefore tried to measure them numerically through single-phase cumulants. We found that the first-order correction to the moments could be obtained to an accuracy of a few per cent from lattices with $L / \xi>5$.

\section{REFERENCES}

1. C. Borgs and R. Kotecký, J. Stat. Phys. 61 (1990) 79.

2. C. Borgs, R. Kotecký and S. Miracle-Solé, J. Stat. Phys. 62 (1991) 529.

3. C. Borgs and W. Janke, Phys. Rev. Lett. 68 (1992) 1738.

4. A. Billoire, R. Lacaze and A. Morel, Nucl. Phys. B370 (1992) 773.

5. J. Lee and J.M. Kosterlitz, Phys. Rev. B43 (1990) 3265.

6. S. Gupta, A. Irbäck and M. Ohlsson, in preparation.

7. K. Binder and D.P. Landau, Phys. Rev. B30 (1984) 1477.

8. M.S. Challa, D.P. Landau and K. Binder, Phys. Rev. B34 (1986) 1841.

9. G.A. Baker Jr and D. Kim, J. Phys. A: Math. Gen. 13 (1980) L103.

Figure 2. Moments $\left\langle m^{k}\right\rangle$ against $L^{-d}$. The curves are explained in the text. 